
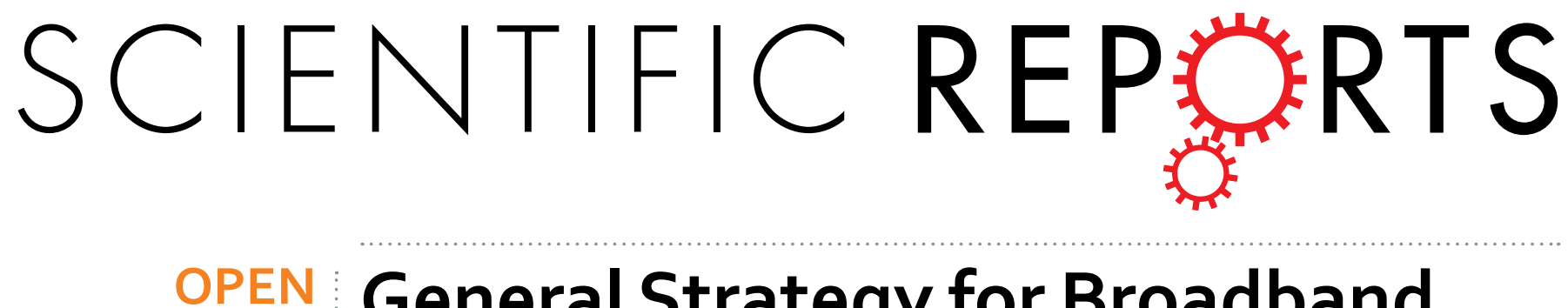

\title{
General Strategy for Broadband Coherent Perfect Absorption and Multi-wavelength All-optical \\ Switching Based on Epsilon-Near- Zero Multilayer Films
}

Received: 07 January 2016

Accepted: 23 February 2016

Published: 11 March 2016
Tae Young Kim ${ }^{1}$, Md. Alamgir Badsha ${ }^{1}$, Junho Yoon ${ }^{1}$, Seon Young Lee ${ }^{1}$, Young Chul Jun ${ }^{2} \&$ Chang Kwon Hwangbo ${ }^{1}$

We propose a general, easy-to-implement scheme for broadband coherent perfect absorption (CPA) using epsilon-near-zero (ENZ) multilayer films. Specifically, we employ indium tin oxide (ITO) as a tunable ENZ material, and theoretically investigate CPA in the near-infrared region. We first derive general CPA conditions using the scattering matrix and the admittance matching methods. Then, by combining these two methods, we extract analytic expressions for all relevant parameters for CPA. Based on this theoretical framework, we proceed to study ENZ CPA in a single layer ITO film and apply it to all-optical switching. Finally, using an ITO multilayer of different ENZ wavelengths, we implement broadband ENZ CPA structures and investigate multi-wavelength all-optical switching in the technologically important telecommunication window. In our design, the admittance matching diagram was employed to graphically extract not only the structural parameters (the film thicknesses and incident angles), but also the input beam parameters (the irradiance ratio and phase difference between two input beams). We find that the multi-wavelength all-optical switching in our broadband ENZ CPA system can be fully controlled by the phase difference between two input beams. The simple but general design principles and analyses in this work can be widely used in various thin-film devices.

Coherent perfect absorption (CPA) occurs due to the interference of two counter-propagating beams, which leads to complete absorption of light in an absorbing medium ${ }^{1-4}$. It is also known as a time-reversed laser or an anti-laser. It was first demonstrated in a Fabry-Perot cavity with a wavelength-scale silicon slab. Incident light is trapped in a cavity, bouncing back and forth, until it is completely absorbed and converted to heat or other form of energy. Later, CPA was studied for various nanostructures and subwavelength thin films ${ }^{5-11}$, and was measured recently for graphene and conducting thin films ${ }^{12,13}$. However, the CPA structures so far are mostly limited to single-frequency operation, and are hard to be extended to a broadband structure - especially in the high, optical frequency region.

Here, we propose a new broadband CPA scheme based on epsilon-near-zero (ENZ) multilayer films. ENZ thin films can be a versatile platform for strong field confinement and for the enhancement of light-matter interactions ${ }^{14-16}$. Recently, it was found that unidirectional perfect absorption can occur in ultra-thin, low-loss ENZ layers ${ }^{17-19}$. Furthermore, at ENZ wavelengths (i.e. $\left.\operatorname{Re}(\varepsilon)=0\right)$, the normal component $\left(\mathrm{E}_{\perp}\right)$ of the electric field can become very strong in a deeply subwavelength film (following the boundary condition $\varepsilon_{1} \mathrm{E}_{1 \perp}=\varepsilon_{2} \mathrm{E}_{2 \perp}$ ). This strong field enhancement in ENZ films was also used to enhance nonlinear harmonic generation or control light absorption electrically in a cavity ${ }^{20,21}$. Interestingly, for very low-loss $(\operatorname{Im}(\varepsilon) \sim 0)$ dielectric films, it was reported that multilayers of high and low index films can be designed for unidirectional perfect absorption with field enhancement $^{22}$. 
(a)

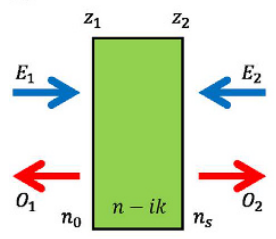

(b)

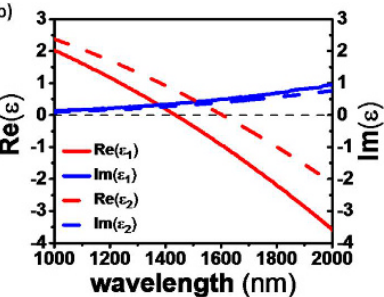

Figure 1. (a) General configuration of a two-port thin film system for CPA. $E_{1}, E_{2}$ and $O_{1}, O_{2}$ are input and output fields, respectively. An absorbing film with a complex refractive index $\left(N_{f}=n-i k\right)$ with thickness $d$ is located between two nonabsorbing media with refractive indices $n_{0}$ and $n_{s}$. (b) The dielectric constants of ITO- 1 and ITO-2 films at near-IR wavelengths. The ENZ wavelengths (i.e. $\operatorname{Re}(\varepsilon)=0)$ are $1435 \mathrm{~nm}$ for ITO-1 and $1605 \mathrm{~nm}$ for ITO-2.

In our previous theoretical and experimental works, we used ITO as a near-infrared (near-IR) ENZ material, and demonstrated perfect absorption in an ultra-thin ITO film. In these works, an ITO film was coated on a reflective substrate (or we worked in the attenuated total reflection condition) in order to suppress the transmission. In this case, by finding the destructive interference condition of reflected light, we could achieve unidirectional perfect absorption (i.e. having a single input beam) ${ }^{23}$. The ENZ wavelengths of ITO films depend on doping levels that can be controlled during the film growth, and thus tunable perfect absorption could be achieved without any structural patterning. Moreover, by using an ITO multilayer of different ENZ wavelengths, we experimentally demonstrated ultra-wideband perfect absorption in the near-IR ${ }^{24}$. Inspired by previous studies, here we propose a general strategy for broadband, bi-directional CPA based on planar, unpatterned films. Again, we use an ITO thin film as an ENZ layer, and find broadband CPA conditions in the near-IR region. Finally, we use this broadband ENZ CPA for multi-wavelength all-optical switching in the telecommunication window. In our analysis, we also fully account for the effects of the substrate. Therefore, we consider realistic configurations for our analysis. This method can be also readily extended to other frequency ranges using different ENZ materials (e.g. it can be extended to mid- and far-infrared using doped semiconductors). Our work shows that ENZ multilayer films provide a very flexible platform for high-frequency, broadband CPA.

In this paper, we first derive the CPA conditions using the scattering matrix and the admittance matching methods separately. Herein, we show that these two CPA analysis methods are equivalent. By combining these two methods, we extract not only the structural parameters of the CPA system, such as thin film thicknesses and incident angles, but also the input beam parameters, such as the amplitude ratio and phase difference between the two input beams. We also show that the broad ENZ wavelength band in the effective dielectric constant of the ITO multilayer film is important in achieving broadband CPA. Finally, we investigate multi-wavelength optical switching in our broadband CPA system consisting of an ITO multilayer sandwiched between two ZnSe prisms with $45^{\circ}$ incident angles. It is also verified that our analytic calculations agree well with numerical simulations. The design principles and analyses used here can be widely applied to various nanophotonic devices and components, such as optical switches, modulators, filters, sensors, and thermal emitters.

\section{Theoretical Framework}

Scattering Matrix Method. We first obtain general conditions for CPA using the scattering matrix method. CPA occurs when the determinant of the scattering matrix of an absorbing medium becomes zero. This is a very general condition that can be applied to either a structured medium or an unpatterned film. The schematic diagram of CPA in an absorbing medium of complex refractive index $N_{f}=n-i k$ (dielectric constant $\varepsilon=N_{f}^{2}$ ) is shown in Fig. 1(a). The monochromatic time harmonic convention, $e^{i \omega t}$, is used in this paper. The optical system consists of two ports; one on the left of the medium and the other on the right. Port 1 has the input and out fields $E_{1}$ and $O_{1}$ in the $+z$ and $-z$ directions, respectively, in the nonabsorbing incident medium of a refractive index $n_{0}$. Similarly, Port 2 has the input and out fields $E_{2}$ and $O_{2}$ in the $-z$ and $+z$ directions, respectively, in the nonabsorbing substrate medium of a refractive index $n_{s}$. The substrate $\left(n_{s}\right)$ and incident $\left(n_{0}\right)$ media are the output media for $E_{1}$ and $E_{2}$, respectively. Then the relationship between the output $\left[\begin{array}{l}O_{1} \\ O_{2}\end{array}\right]$ and input $\left[\begin{array}{l}E_{1} \\ E_{2}\end{array}\right]$ fields can be expressed using a scattering matrix as

$$
\left[\begin{array}{l}
O_{1} \\
O_{2}
\end{array}\right]=[S]\left[\begin{array}{l}
E_{1} \\
E_{2}
\end{array}\right]
$$

where the scattering matrix $[S]$ is defined as

$$
[S]=\left[\begin{array}{ll}
\rho_{1} & \tau_{2} \\
\tau_{1} & \rho_{2}
\end{array}\right]
$$

The elements of the scattering matrix are as follows: $\rho_{1}=\left|\rho_{1}\right| e^{i \varphi_{\rho 1}}$ and $\tau_{1}=\left|\tau_{1}\right| e^{i \varphi_{\tau 1}}$ are the reflection and transmission coefficients, respectively, of the incident field $E_{1}$, and $\rho_{2}=\left|\rho_{2}\right| e^{i \varphi_{\rho 2}}$ and $\tau_{2}=\left|\tau_{2}\right| e^{i \varphi_{\tau 2}}$ are the reflec- 
tion and transmission coefficients, respectively, of the incident field $E_{2}{ }^{25,26}$. The reflection phases are $\varphi_{\rho 1}$ and $\varphi_{\rho 2}$, and the transmission phases are $\varphi_{\tau 1}$ and $\varphi_{\tau 2}$.

Since CPA occurs when $\mathrm{O}_{1}=\mathrm{O}_{2}=0$ (i.e. the determinant of scattering matrix [S] must be zero), the CPA condition is given by

$$
\rho_{1} \rho_{2}=\tau_{1} \tau_{2}
$$

This can be, in turn, rewritten as

$$
R_{1} R_{2}=T^{2}
$$

and

$$
\varphi_{\tau}=\frac{1}{2}\left(\varphi_{\rho 1}+\varphi_{\rho 2}\right) \text { or } \frac{1}{2}\left(\varphi_{\rho 1}+\varphi_{\rho 2}\right)+\pi,
$$

where $R_{1}=\left|\rho_{1}\right|^{2}$ and $R_{2}=\left|\rho_{2}\right|^{2}$. In deriving Eqs (4) and (5), the principle of the reversibility of $T=\frac{\eta_{s}}{\eta_{0}}\left|\tau_{1}\right|^{2}=\frac{\eta_{0}}{\eta_{s}}\left|\tau_{2}\right|^{2}$ and $\varphi_{\tau 1}=\varphi_{\tau 2}$ were employed. The definitions of the admittances $\eta_{0}, \eta_{f}$, and $\eta_{s}$ in each medium for the TE and TM waves are presented in Supplementary Information (Supplementary Note A) ${ }^{26,27}$.

By taking into account the amplitude ratio and phase difference between the two input beams, Eq. (1) can be expressed as

$$
\left[\begin{array}{l}
O_{1} \\
O_{2}
\end{array}\right]=\left[\begin{array}{ll}
\rho_{1} & \tau_{2} \\
\tau_{1} & \rho_{2}
\end{array}\right]\left[\sqrt{\frac{\eta_{0}}{\eta_{s}} \frac{1}{P_{i n 2}}} e^{-i \varphi_{12}}\right]\left|E_{1}\right|,
$$

where $\varphi_{12}=\varphi_{2}-\varphi_{1}$ is the phase difference between the two input beams, and the input irradiances at Port 1 and 2 are defined respectively as $P_{i n 1}=\frac{1}{2} y_{0} \eta_{0}\left|E_{1}\right|^{2}$ and $P_{i n 2}=\frac{1}{2} y_{0} \eta_{0}\left|E_{2}\right|^{2}$, where $y_{0}=\sqrt{\frac{\varepsilon_{0}}{\mu_{0}}}$ is the admittance of the vacuum. Then, the output irradiance $P_{\text {out } 1}$ in the incident medium of Port 1 can be written as

$$
P_{\text {out } 1}=\frac{1}{2} y_{0} \eta_{0}\left|O_{1}\right|^{2}=\frac{1}{2} y_{0} \eta_{0}\left[R_{1}+\frac{P_{i n 2}}{P_{i n 1}} T+2 \sqrt{\frac{P_{i n 2}}{P_{i n 1}}} \sqrt{R_{1} T} \cos \Delta_{1}\right],
$$

where $\Delta_{1}=\varphi_{\rho 1}-\varphi_{\tau}+\varphi_{12}$ is the interference phase, and we assumed $\left|E_{1}\right|=1$. Similarly, the output irradiance $P_{\text {out } 2}$ in the substrate becomes

$$
P_{\text {out } 2}=\frac{1}{2} y_{0} \eta_{s}\left|O_{2}\right|^{2}=\frac{1}{2} y_{0} \eta_{0}\left[T+\frac{P_{\text {in } 2}}{P_{\text {in } 1}} R_{2}+2 \sqrt{\frac{P_{\text {in } 2}}{P_{\text {in } 1}}} \sqrt{R_{2} T} \cos \Delta_{2}\right]
$$

where $\Delta_{2}=\varphi_{\rho_{2}}-\varphi_{\tau}-\varphi_{12}$.

Since $P_{\text {out } 1}=P_{\text {out } 2}=0$ at CPA (i.e. complete destructive interference) occurs in Eqs (7) and (8), the input beam requirements, namely, the irradiance ratio and phase shift, can be respectively derived to be

$$
\frac{P_{i n 2}}{P_{i n 1}}=\frac{R_{1}}{T}=\frac{T}{R_{2}}
$$

and

$$
\varphi_{12}=\frac{1}{2}\left(\varphi_{\rho 2}-\varphi_{\rho 1}\right) \text { or } \frac{1}{2}\left(\varphi_{\rho 2}-\varphi_{\rho 1}\right)+\pi .
$$

Equation (9), which is equivalent to Eq. (4), is related to balancing of the amplitudes between two output beams. Then, the output irradiances become

$$
P_{\text {out } 1}=y_{0} \eta_{0} R_{1}\left(1+\cos \Delta_{1}\right)
$$

and

$$
P_{\text {out } 2}=y_{0} \eta_{0} T\left(1+\cos \Delta_{2}\right) .
$$

If $\varphi_{\rho 2}=\varphi_{\rho 1}$ or $\varphi_{\rho 1}+\pi$ in Eq. (10) (i.e., if $\varphi_{12}=0$ or $\pi$ ), then $P_{\text {out } 1}=P_{\text {out } 2}=0$, which is "two-port" CPA. If $\varphi_{12}=0$, the two input beams are called symmetric, whereas if $\varphi_{12}=\pi$, they are antisymmetric. Equations (11) and (12) indicate that CPA can be used as a sinusoidal optical switch or modulator if $\varphi_{12}$ is varied. We may tune $\varphi_{12}$ to make either $P_{\text {out } 1}=0$ or $P_{\text {out } 2}=0$, yielding "one-port" CPA. The output irradiances and absorptance $(A)$ in the figures presented herein were normalized with respect to the total input irradiance $\left(P_{i n 1}+P_{i n 2}\right)$ : $\frac{P_{\text {out } 1}}{P_{\text {in } 1}+P_{\text {in } 2}} \rightarrow P_{\text {out } 1}, \quad \frac{P_{\text {out } 2}}{P_{\text {in } 1}+P_{\text {in } 2}} \rightarrow P_{\text {out } 2}, \quad$ and $A=1-\frac{P_{\text {out } 1}+P_{\text {out } 2}}{P_{\text {in } 1}+P_{\text {in } 2}}$. 
Admittance Matching Method. Now we consider CPA conditions using the admittance matching method that can be applied to unpatterned films. This will be eventually used in the next section when we design a near-IR CPA system with ITO multilayers.

When CPA occurs with $O_{1}=O_{2}=0$ in Fig. 1, only one incident beam exists in each medium: $E_{1}$ in the incident medium $\left(n_{0}\right)$ and $E_{2}$ in the substrate $\left(n_{s}\right)$. Since the waves in the two media are counter-propagating, the magnetic field direction in the substrate should be reversed in the opposite direction to that in the incident medium (i.e., $H \rightarrow-H)$ if the electric field direction is the same. Then the admittance of the substrate can be defined as $-\eta_{s}$ when CPA is present ${ }^{28}$. The negative admittance does not mean a negative index.

Therefore the forward admittance of the film starting from $\left(-\eta_{s}, 0\right)$ can be calculated using the transfer matrix method (TMM) as follows:

$$
\left[\begin{array}{l}
B \\
C
\end{array}\right]=\left[\begin{array}{lc}
\cos \delta & i \frac{\sin \delta}{\eta_{f}} \\
i \eta_{f} \sin \delta & \cos \delta
\end{array}\right]\left[\begin{array}{c}
1 \\
-\eta_{s}
\end{array}\right],
$$

where $B$ and $C$ are the normalized electric and magnetic fields, respectively, in the incident medium, and the optical phase thickness is defined as $\delta=\frac{2 \pi d}{\lambda} N_{f} \cos \theta$. And $N_{f}=n-i k, d$, and $\eta_{f}$ are the complex refractive index of the film, physical thickness, and admittance of the thin film, respectively ${ }^{26}$. Snell's law is valid here: $n_{0} \sin \theta_{0}=(n-i k) \sin \theta=n_{s} \sin \theta_{s}$, where $\theta_{0}, \theta$, and $\theta_{s}$ are the incident angle, transmitted angle in the film, and transmitted angle in the substrate, respectively. The surface admittance of the film at $z_{1}$ is given by

$$
Y=\frac{C}{B} \text {. }
$$

Then, CPA occurs when

$$
Y=\eta_{0}
$$

where $\eta_{0}$ is the admittance of the incident medium. From Eqs (13) (15), the CPA condition for an absorbing thin-film layer can be derived to be

$$
\tan \delta=-i \frac{\eta_{f}\left(\eta_{0}+\eta_{s}\right)}{\eta_{f}^{2}+\eta_{0} \eta_{s}}
$$

If the backward admittance matching is traced from $\eta_{0}$ to $-\eta_{s}$ using $-\delta$ in Eq. (13), the same CPA condition as Eq. (16) can be obtained. For a free-standing film in air at normal incidence, Eq. (16) is identical to Eqs (4, 7 and 14) in Refs 1,7 and 8, respectively.

The structural parameters of thin films exhibiting CPA, such as their materials, thicknesses, and the incident light angles in the two media, can be determined from Eq. (16). They can be also obtained graphically using the admittance diagram ${ }^{23,28}$. In the next section, we will use the admittance diagram to determine the CPA parameters for ITO thin films. The elements of the scattering matrix in Eq. (2) are obtained in Supplementary Note A. It is also shown therein that Eq. (16), which was derived using admittance matching, is equivalent to Eq. (3), obtained from the scattering matrix. Thus, the relationship between the reflection and transmission coefficients can be expressed as

$$
\rho_{2}=\frac{\eta_{0}\left(\eta_{s}^{2}-\eta_{f}^{2}\right)}{\eta_{s}\left(\eta_{0}^{2}-\eta_{f}^{2}\right)} \rho_{1}
$$

and

$$
\tau_{2}=\frac{\eta_{s}}{\eta_{0}} \tau_{1} .
$$

Then, the CPA condition of Eq. (3) for a single layer can be expressed as

$$
\left(\rho_{1}-\sqrt{\frac{\eta_{0}^{2}-\eta_{f}^{2}}{\eta_{s}^{2}-\eta_{f}^{2}}} \tau_{2}\right)\left(\rho_{1}+\sqrt{\frac{\eta_{0}^{2}-\eta_{f}^{2}}{\eta_{s}^{2}-\eta_{f}^{2}}} \tau_{2}\right)=0,
$$

indicating that two input beams have even and odd phases. Also, the input beam parameters for single-layer CPA (more specifically, the irradiance ratio and phase shift given in Eqs (9) and (10)) can be respectively expressed by

$$
\frac{P_{i n 2}}{P_{i n 1}}=\left(\frac{\eta_{s}}{\eta_{0}}\right)\left|\frac{\eta_{0}^{2}-\eta_{f}^{2}}{\eta_{s}^{2}-\eta_{f}^{2}}\right|
$$

and 


$$
\varphi_{12}=0 \text { or } \pi \text {. }
$$

If a single absorbing layer cannot complete this admittance matching between the substrate and the incident medium, another phase-matching dielectric layer can be added to complete the trajectory of the admittance locus for Eq. (15) in the admittance matching diagram, in which case the phase shift of Eq. (21) is not necessarily 0 or $\pi^{28}$.

In our previous work on unidirectional perfect absorption, we showed that the admittance matching condition (Eq. (15)) can be satisfied in an ultra-thin, low-loss ENZ film for p-polarized oblique incidence of light ${ }^{23,24}$. This can be directly extended to bi-directional CPA based on ENZ films, using the negative admittance for a substrate. Moreover, employing an ENZ multilayer film, we can achieve broadband CPA. This will be discussed more in the next section using ITO films.

ENZ CPA can be also understood as critical coupling, as explained in Supplementary Notes B and C. Since ENZ CPA is caused by resonant plasmon absorption, we can rewrite the admittance matching condition of Eq. (16) as the phase matching condition for transverse resonance in the film:

$$
2 \kappa_{f} d=2 \varphi_{0}+2 \varphi_{s}+2 m \pi,
$$

where $\kappa_{f}$ is the transverse wavevector in the film, $2 \varphi_{0}$ and $2 \varphi_{s}$ are the reflection phases at the incident medium-film and film-substrate interfaces, respectively, and $m$ is the order of a resonance mode. Eq. (22) indicates that once the critical coupling condition is satisfied for an ENZ film, two counter-propagating waves in the absorbing film interfere constructively, behave like an inhomogeneous guided mode in the film, and are totally absorbed as the mode propagates in the film.

\section{Results}

The ENZ wavelengths of ITO films in the near-IR region can be controlled by doping levels. In our previous work, broadband perfect absorption with unidirectional illumination was experimentally realized using an ITO multilayer film ${ }^{24}$. In this work, we again use ITO as a tunable ENZ material. More specifically, we use two ITO films (ITO-1, ITO-2) as near-IR ENZ materials, whose ENZ wavelengths (i.e. $\operatorname{Re}(\varepsilon)=0)$ are $1435 \mathrm{~nm}(\mathrm{ITO}-1)$ and $1605 \mathrm{~nm}$ (ITO-2), as shown in Fig. 1(b) ${ }^{29}$.

ENZ CPA in a Single-layer ITO Thin Film and Its Application to All-optical Switching. We start with a CPA structure including a single ITO layer (ITO-1). We assume different materials for the incident and substrate media (i.e. an asymmetric structure), as shown in Fig. 2(a). We will first find CPA parameters graphically using the admittance matching diagram ${ }^{26,28,30}$, and then compare them with the general CPA conditions derived from the scattering matrix method.

The admittance diagram in Fig. 2(b) shows the admittance matching results for [Glass|ITO-1|ZnSe] at a wavelength of $1424 \mathrm{~nm}$; the forward locus of the modified admittance of the ENZ ITO film starts at the negative modified admittance of $(-1.05,0)$ for the $\mathrm{ZnSe}$ substrate, makes a large circle in the clockwise direction corresponding to the film thickness of $23.13 \mathrm{~nm}$, and arrives at a positive modified admittance of $(1.50,0)$ for the glass ${ }^{28}$. The incident angles were determined to be $70^{\circ}$ in the glass and $35.2^{\circ}$ in the $\mathrm{ZnSe}$ substrate.

The absorption spectrum of [Glassr|ITO-1|ZnSe] is calculated using the transfer matrix method (TMM), and is shown in Fig. 2(c), revealing CPA $(A=1)$ at $1424 \mathrm{~nm}$, which is slightly shorter than the ENZ wavelength of $1435 \mathrm{~nm}$. The same behavior was also observed in our previous study on unidirectional perfection absorption in ITO films ${ }^{23}$. It can be understood in this way: since CPA occurs due to critical coupling to a radiative mode in the ENZ thin film, CPA should follow the dispersion of this mode, which lies slightly above the ENZ frequency $(\operatorname{Re}(\varepsilon)=0)$. Therefore, CPA occurs at a slightly shorter wavelength than the ENZ wavelength.

The elements of the scattering matrix in Eq. (2) were also calculated in Fig. 2(d). It can be seen that $T=\sqrt{R_{1} R_{2}}$ (red) occurs at $1424 \mathrm{~nm}$ and $1446 \mathrm{~nm}$ and that $\left[\varphi_{t}=\frac{1}{2}\left(\varphi_{\rho 1}+\varphi_{\rho 2}\right)\right]$ (blue) occurs at $1424 \mathrm{~nm}$. The CPA wavelength at $1424 \mathrm{~nm}$, which satisfies both Eqs (4) and (5) and is shown in the inset of Fig. 2(d), was also used to obtain the admittance matching in Fig. 2(b), implying that Eq. (16) is equivalent to Eq. (3) for the single-layer $\mathrm{CPA}$. We obtained also the input beam parameters; the irradiance ratio and phase shift between the two input beams were determined from Eqs (9) and (10) to be $\frac{P_{i n 2}}{P_{i n 1}}=1.47$ and $\varphi_{12}=0^{\circ}$, respectively.

If we apply Eq. (22) for this ENZ CPA at $1424 \mathrm{~nm}$ (TM wave), we obtain the subwavelength waveguide characteristics of ENZ CPA as follows; the vertical wavevector of ENZ CPA is $\kappa_{f}=(0.55-i 6.15) \times 10^{6} \mathrm{~m}^{-1}$, the order is $m=0$, and the effective index of a propagating ENZ CPA wave is $N_{e f f}=1.41$. We find that the electric field decreases rapidly in the transverse direction, and the ultrathin subwavelength thickness obtained from the admittance matching of Eq. (16) is in good agreement with the zeroth order of Eq. (22), implying that ENZ CPA occurs due to critical coupling to a propagating wave in the longitudinal direction along the film.

Now, we use this ENZ CPA for all-optical switching. The 2D contour maps of $P_{\text {out } 1}$ and $P_{\text {out } 2}$ are shown as functions of the relative phase shift $\varphi_{12}$ and the incident wavelength in Fig. 3. Firstly, two-port CPA (i.e., $O_{1}=O_{2}=0$ ) is observed at $\lambda=1424 \mathrm{~nm}, \varphi_{12}=0^{\circ}$ in both Fig. 3(a,b). Secondly, one-port CPA with $O_{1}=0$ occurs at $\lambda=1446 \mathrm{~nm}, \varphi_{12}=-16^{\circ}$ in Fig. 3(a), whereas one-port CPA with $O_{2}=0$ at $\lambda=1446 \mathrm{~nm}, \varphi_{12}=24^{\circ}$ in Fig. 3(b). The one-port CPA at $1446 \mathrm{~nm}$ can be explained as follows: Though the output irradiances are given by Eqs (11) and (12) due to $T=\sqrt{R_{1} R_{2}}$ at $1446 \mathrm{~nm}$ as shown in Fig. 3(d), the phase change is either $\Delta_{1}=0^{\circ}$ or $\Delta_{2}=0^{\circ}$ depending on how the external phase shift $\varphi_{12}$ is controlled.

The two-port CPA optical switch at $1424 \mathrm{~nm}$ is shown as a function of the phase shift $\varphi_{12}$ in Fig. 3(c): the "ON" state $\left(O_{1}=O_{2}=0\right)$ is apparent at $\varphi_{12}=0^{\circ}$, and the optical switching effects are same in both ports. Optical switching behaviors at $1446 \mathrm{~nm}$ are also evident in Fig. 3(d). At $\varphi_{12}=-16^{\circ}$ the "ON" state $\left(O_{1}=0\right)$ appears in 
(a)

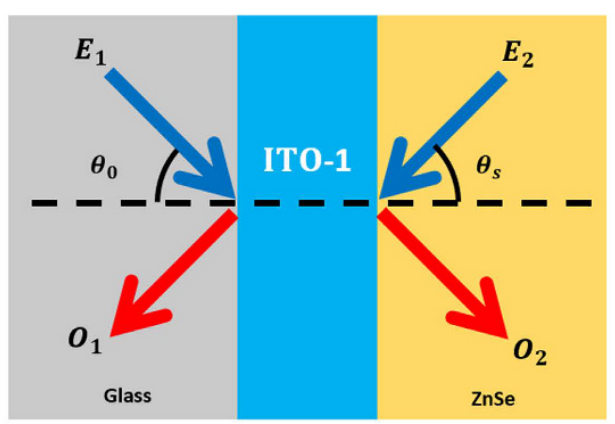

(c)

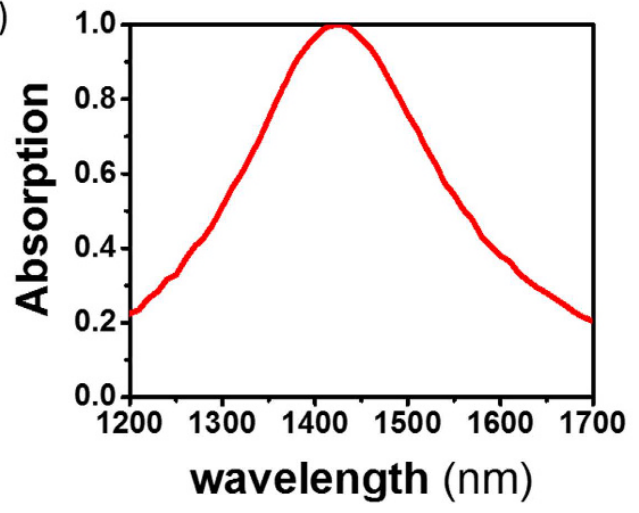

(b)
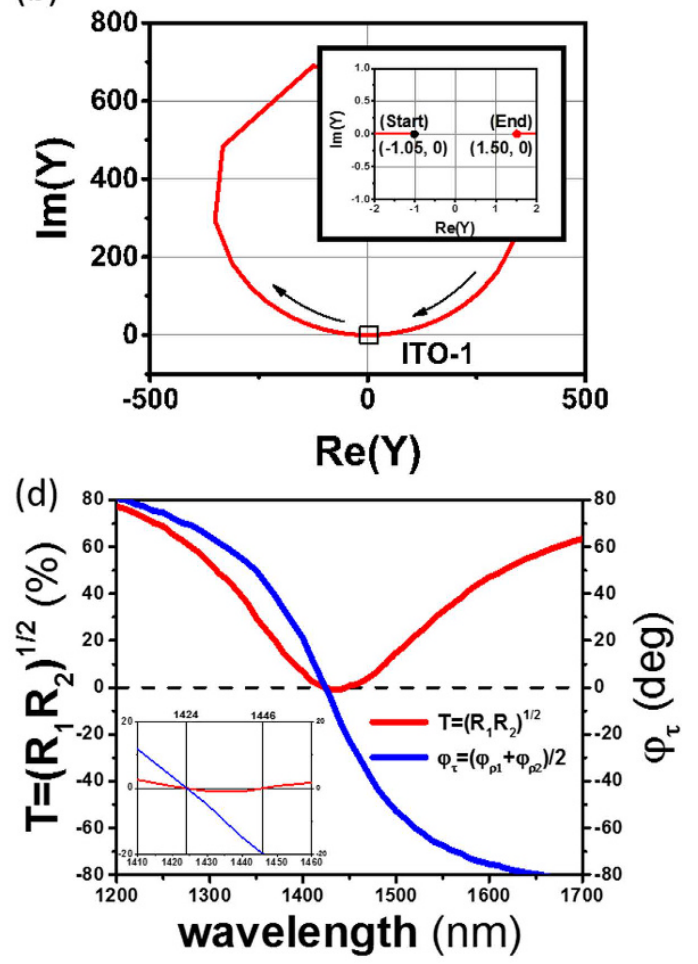

Figure 2. (a) Schematic diagram of ENZ ITO thin-film [Glass $\mid$ ITO-1|ZnSe] CPA device; (b) the modified admittance diagram of [Glass|ITO-1|ZnSe] at $1424 \mathrm{~nm}$ wavelength, at oblique angles of $70^{\circ}$ in glass $\left(n_{0}=1.50\right)$ and $35.2^{\circ}$ in $\mathrm{ZnSe}\left(n_{s}=2.45\right)$, and at $23.13 \mathrm{~nm}$ ITO- 1 film thickness $\left(N_{f}=0.458-i 0.382\right)$ (inset shows start and end admittances); (c) calculated CPA spectrum of [Glass|ITO-1|ZnSe], $A=1$ (CPA) is observed at $1424 \mathrm{~nm}$; (d) calculated $\left(T-\sqrt{R_{1} R_{2}}\right)$ (red) and $\left[\varphi_{t}-\frac{1}{2}\left(\varphi_{\rho 1}+\varphi_{\rho 2}\right)\right]$ (blue) of [Glass|ITO-1|ZnSe] as a function of wavelength (inset is expanded view near CPA wavelength regime); $T-\sqrt{R_{1} R_{2}}$ occurs at 1424 and $1446 \mathrm{~nm}$ whereas $\varphi_{t}=\frac{1}{2}\left(\varphi_{\rho 1}+\varphi_{\rho 2}\right)$ occurs at $1424 \mathrm{~nm}$.

Port 1 , whereas the "OFF" state $\left(O_{2} \neq 0\right)$ occurs in Port 2 , while at $\varphi_{12}=24^{\circ}$ the optical switch state is reversed. Equation (4) has two solutions at different wavelengths: one for two-port CPA and the other for one-port CPA. The optical switching phenomena in [Glass|ITO-1|ZnSe] CPA are summarized in Table 1.

Broadband ENZ CPA in ITO Multilayers. Extending the previous ITO single-layer study, we now propose a broadband ENZ CPA system and apply it to multi-wavelength all-optical switching. As shown in Fig. 4(a), we consider an ITO double layer sandwiched between two ZnSe prisms (i.e. [ZnSe|ITO-1|ITO-2|ZnSe]), and fix the incidence angles as $45^{\circ}$ in the both $\mathrm{ZnSe}$ prisms $\left(n_{0}=n_{s}=2.45\right)$. This configuration was chosen to realize near-IR broadband CPA while keeping the structure as simple as possible. We can broaden the bandwidth further by increasing the number of ITO layers. The ENZ wavelengths (i.e. $\operatorname{Re}(\varepsilon)=0$ ) of each ITO film are $1435 \mathrm{~nm}$ for ITO-1 and $1605 \mathrm{~nm}$ for ITO-2 (Fig. 1(b)). As we did in the previous section, we will first find CPA parameters graphically using the admittance matching diagram, and then compare them with the general CPA conditions derived from the scattering matrix method.

The admittance diagram shows that the forward modified admittance locus of ITO-1 (blue line) at a TM-wave wavelength of $1550 \mathrm{~nm}$ starts at $(-2.45,0)$ in Fig. 4(b), while the backward modified admittance (red line) begins at $(+2.45,0)$. The intersection of two loci of ITO- 1 and ITO- 2 at $(-1.35,-2.05)$ indicates ITO- 1 and ITO- 2 thicknesses of $14.22 \mathrm{~nm}$ and $20.13 \mathrm{~nm}$, respectively. Furthermore, the irradiance ratio and phase shift between the two input beams were determined from Eqs (9) and (10) as $\frac{P_{i n 2}}{P_{i n}}=1$ and $\varphi_{12}=1^{\circ}$, respectively. This method is a simple and intuitive means of determining the structural parameters in Eq. (15) for ENZ thin films exhibiting $\mathrm{CPA}$, such as the thin-film materials, thicknesses, and the incident angles in media.

The normalized output irradiances $\log \left(P_{\text {out } 1}\right)$ and $\log \left(P_{\text {out } 2}\right)$ were calculated and are shown in Fig. 5(a). The broadband range of CPA with $>99 \%$ absorptance is apparent in $1443 \sim 1576 \mathrm{~nm}$, and two CPA dips are evident at $1466 \mathrm{~nm}$ and $1550 \mathrm{~nm}$ in the CPA band. The CPA conditions were calculated using Eqs (4) and (5) and are plotted as a function of wavelength in Fig. 5(b). Four wavelengths with $T=\sqrt{R_{1} R_{2}}$ are evident: $1381 \mathrm{~nm}, 1463 \mathrm{~nm}$, $1550 \mathrm{~nm}$, and $1683 \mathrm{~nm}$, and three wavelengths with $\varphi_{t}=\frac{1}{2}\left(\varphi_{\rho 1}+\varphi_{\rho 2}\right)$ are apparent: $1474 \mathrm{~nm}, 1497 \mathrm{~nm}$, and $1550 \mathrm{~nm}$. The band with $T \approx \sqrt{R_{1} R_{2}}$ and $\varphi_{t}=\frac{1}{2}\left(\varphi_{\rho 1}+\varphi_{\rho 2}\right)$ is between 1443 and $1576 \mathrm{~nm}$, which is consistent with the broad CPA band shown in Fig. 5(a). In addition, the CPA wavelength satisfying both CPA conditions simultaneously was found to be $1550 \mathrm{~nm}$; perfect admittance matching occurs at this wavelength, as shown in Fig. 5(b). 
(a)

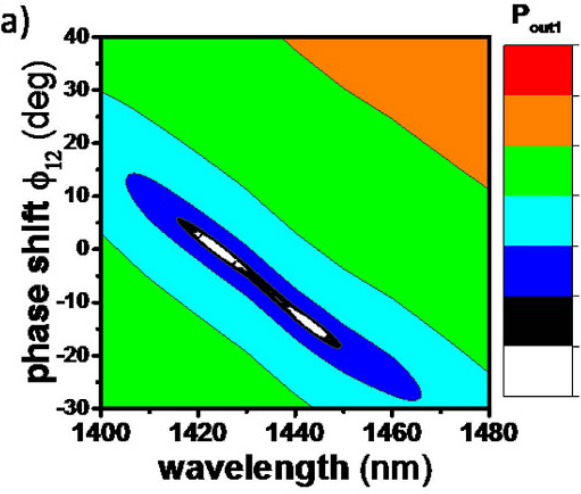

(c)

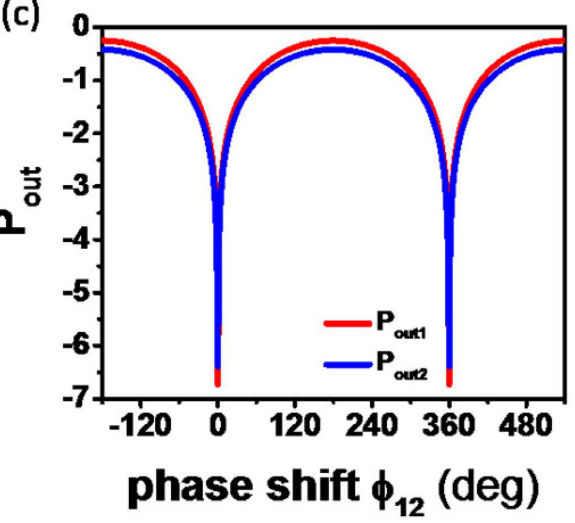

(b)

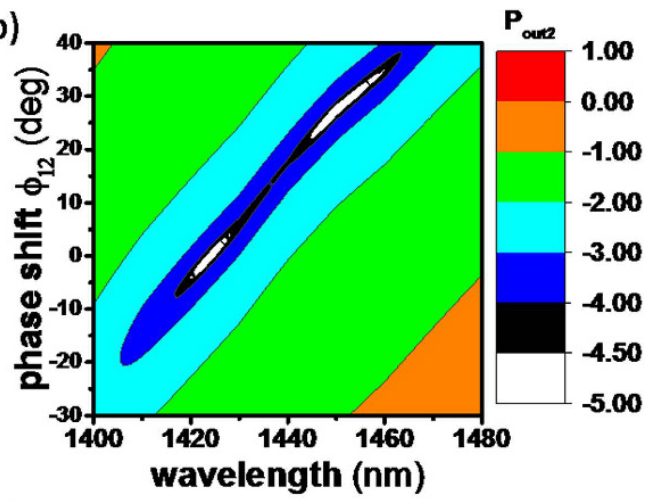

(d)

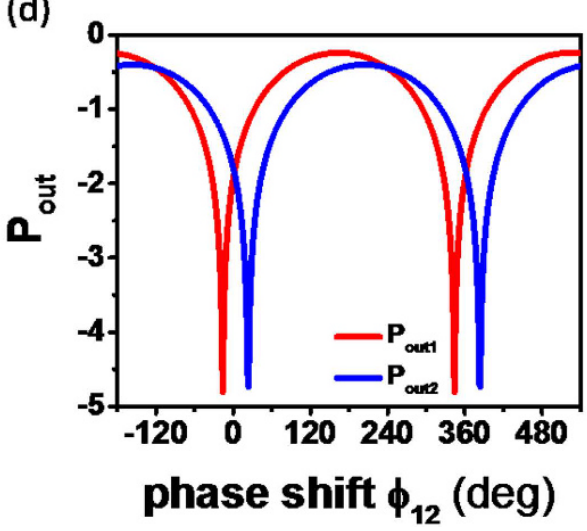

Figure 3. $2 \mathrm{D}$ contour maps of $(\mathbf{a}) \log \left(P_{\text {out } 1}\right)$ and $(\mathbf{b}) \log \left(P_{\text {out } 2}\right)$ as functions of $\varphi_{12}$ and wavelength. The color map is on the $\log$ scale. CPA occurs at $1424 \mathrm{~nm}, 0^{\circ}$ and $1446 \mathrm{~nm},-26^{\circ}$ in $P_{\text {out } 1}$ and $1424 \mathrm{~nm}, 0^{\circ}$ and $1446 \mathrm{~nm}, 24^{\circ}$ in $P_{\text {out } 2}$. Calculated optical modulations of $P_{\text {out } 1}$ and $P_{\text {out } 2}$ on the $\log$ scale as a function of $\varphi_{12}$ at wavelengths of $(\mathbf{c})$ $1424 \mathrm{~nm}$ and (d) $1446 \mathrm{~nm}$.

\begin{tabular}{|c|c|c|c|c|}
\hline & $\begin{array}{c}\text { Wavelength } \\
(\mathrm{nm})\end{array}$ & $\begin{array}{l}\text { Phase shift } \\
\left(\phi_{12}, \text { degree }\right)\end{array}$ & $P_{\text {out } 1}($ Port 1) & $P_{\text {out } 2}($ Port 2) \\
\hline \multirow{3}{*}{ Single- wavelength CPA } & 1424 & 0 & $\begin{array}{c}\text { ON } \\
\left(P_{\text {out } 1}=0, \Delta_{1}=0^{\circ}\right)\end{array}$ & $\begin{array}{c}\text { ON } \\
\left(P_{\text {out } 2}=0, \Delta_{2}=0^{\circ}\right)\end{array}$ \\
\hline & 1446 & -16 & $\begin{array}{c}\text { ON } \\
\left(P_{\text {out } 1}=0, \Delta_{1}=0^{\circ}\right)\end{array}$ & $\begin{array}{c}\text { OFF } \\
\left(P_{\text {out } 2}=0.05, \Delta_{2}=40^{\circ}\right)\end{array}$ \\
\hline & & 24 & $\begin{array}{c}\text { OFF } \\
\left(P_{\text {out } 1}=0.07, \Delta_{1}=40^{\circ}\right)\end{array}$ & $\begin{array}{c}\text { ON } \\
\left(P_{\text {out } 2}=0, \Delta_{2}=0^{\circ}\right)\end{array}$ \\
\hline
\end{tabular}

Table 1. Multi-wavelength optical switching in [Glass|ITO-1|ZnSe]. If $P_{\text {out }}=0$, the switch is defined as "ON."

(a)

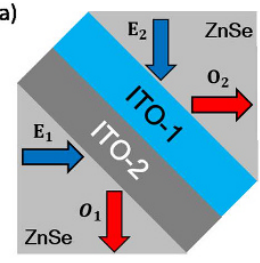

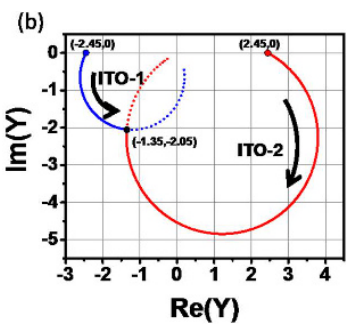

Figure 4. (a) Schematic diagram of broadband CPA device [ZnSe|ITO-1|ITO-2|ZnSe] and (b) modified admittance diagram of [ZnSe|ITO-1(14.22nm)|ITO-2(20.13 nm)|ZnSe] at $1550 \mathrm{~nm}$ in wavelength and with $45^{\circ}$ incident angles, where $N_{I T O-1}=0.27-i 0.84, N_{I T O-2}=0.59-i 0.30$, and $n_{Z n S e}=2.45$ were used.

Since the thicknesses of the ITO-1 and ITO-2 layers are much less than the wavelength $\left(\frac{d}{\lambda}<\frac{1}{50}\right)$, the effective dielectric constant $\left(\varepsilon_{\text {eff }}\right)$ of the ITO double layer can be calculated using the effective medium approximation $^{24,31}$ : 

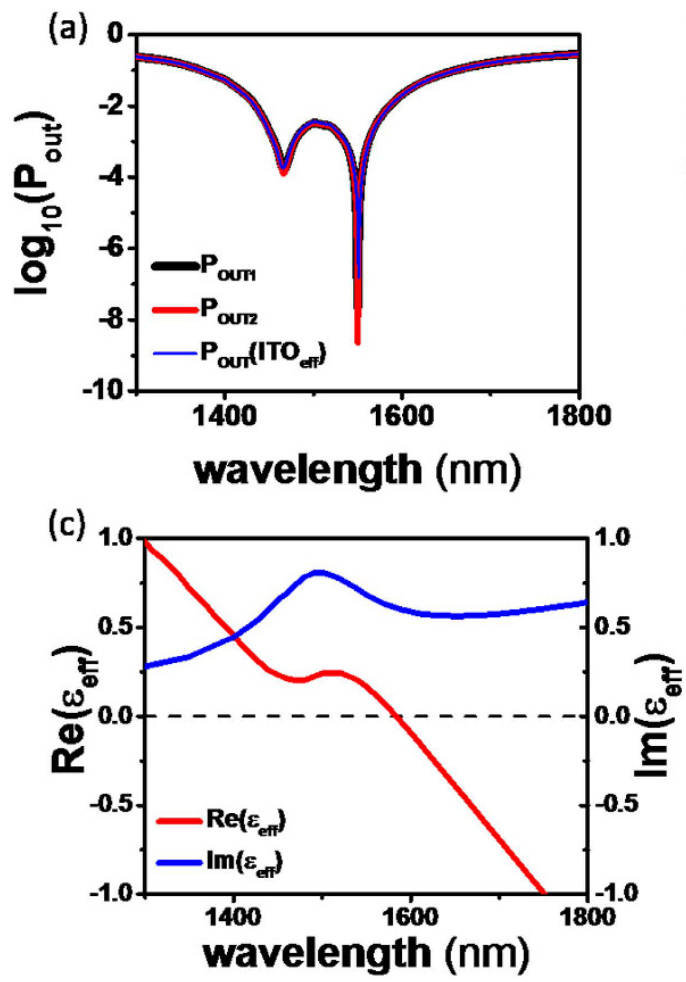

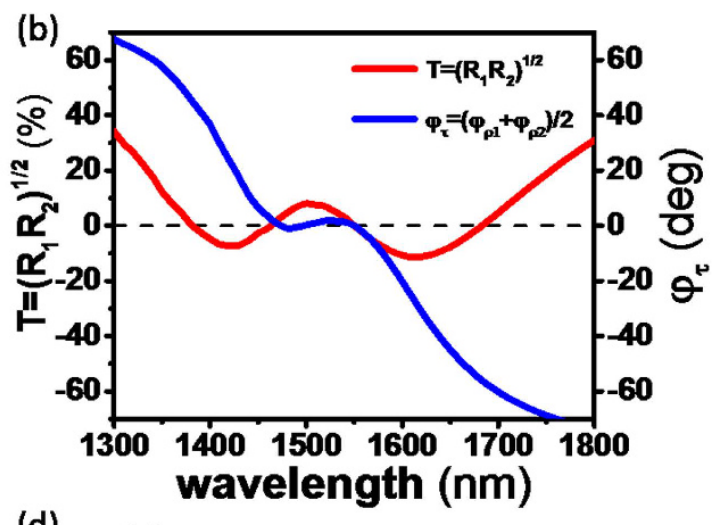

(d)

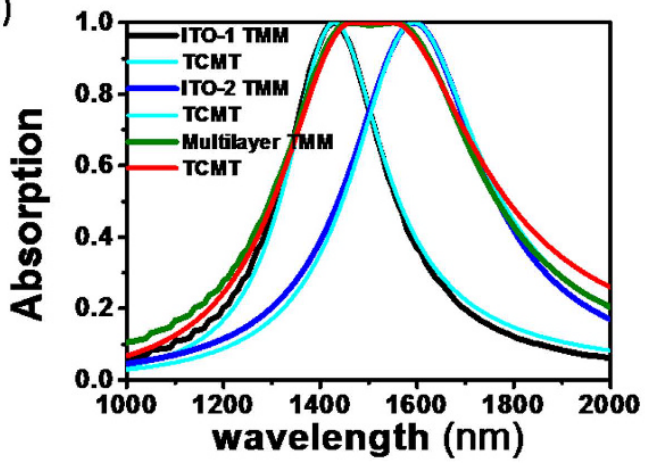

Figure 5. (a) Calculated output irradiances $\log \left(P_{\text {out } 1}\right)$ and $\log \left(P_{\text {out } 2}\right)$ of $[\mathrm{ZnSe}|\mathrm{ITO}-1| \mathrm{ITO}-2 \mid \mathrm{ZnSe}]$ at near IR wavelengths. $\log \left(P_{\text {out } 1}\right)$ and $\log \left(P_{\text {out } 2}\right)$ of $[\mathrm{ZnSe} \mid$ Effective ITO $\mid \mathrm{ZnSe}]$ were also calculated. (b) Calculated $\left(T-\sqrt{R_{1} R_{2}}\right)$ (red) and $\left[\varphi_{t}-\frac{1}{2}\left(\varphi_{\rho 1}+\varphi_{\rho 2}\right)\right]$ (blue) of [ZnSe|ITO-1|ITO-2|ZnSe] as a function of wavelength; $T-\sqrt{R_{1} R_{2}}$ occurs at $1381 \mathrm{~nm}, 1463 \mathrm{~nm}, 1550 \mathrm{~nm}$, and $1683 \mathrm{~nm}$, whereas $\varphi_{t}-\frac{1}{2}\left(\varphi_{\rho 1}+\varphi_{\rho 2}\right)$ occurs at $1474 \mathrm{~nm}$, $1497 \mathrm{~nm}$, and $1550 \mathrm{~nm}$. (c) $\varepsilon_{\text {eff }}$ of ITO-1 and ITO-2 layers calculated by using effective medium approximation. ENZ wavelength at $\operatorname{Re}\left(\varepsilon_{e f f}\right)=0$ is $1585 \mathrm{~nm}$, and the broadband ENZ regime is evident at $1443-1576 \mathrm{~nm}$. (d) Absorption spectra of [ZnSe|ITO-1(14.22nm)|ZnSe] and [ZnSe|ITO-2(20.13nm)|ZnSe] calculated by TMM and fitted by Lorentzian CPA spectrum of Eq. (S15 using TCMT in Supplementary Note B. Broad CPA spectrum of [ZnSe|ITO-1|ITO-2|ZnSe] calculated by TMM was also fitted by equivalent CPA spectrum using Eq. (S15) and two Lorentzian spectra.

$$
\frac{1}{\varepsilon_{e f f}}=\frac{f}{\varepsilon_{1}}+\frac{1-f}{\varepsilon_{2}},
$$

where $f$ is the ratio of ITO-1 layer thickness to the total thickness. Figure 5(c) shows the real and imaginary parts of $\varepsilon_{e f f}$ for the two ENZ ITO layers. Its real part $\operatorname{Re}\left(\varepsilon_{\text {eff }}\right)$ is close to zero over the broad spectral region from $1443-1576 \mathrm{~nm}$, indicating that the broadband ENZ regime was obtained. Using $\varepsilon_{e f f}$, the CPA spectrum was calculated, and Fig. 5(d) shows that the broad ENZ wavelength region corresponds closely to the broad CPA regime. Again, broad CPA (i.e., radiative mode) occurs at broad $\operatorname{Re}\left(\varepsilon_{\text {eff }}\right)$ slightly larger than zero and at shorter wavelength regime than the ENZ wavelength $(1585 \mathrm{~nm})$ of the effective layer at $\operatorname{Re}\left(\varepsilon_{\text {eff }}\right)=0$.

Since ENZ absorption is caused by radiative resonant plasmons, Eq. (S23) of TCMT in Supplementary Note $\mathrm{D}$ can be applied to determining the damping constants of ENZ resonances at CPA. The absorption spectra of [ZnSe|ITO-1 $(14.22 \mathrm{~nm}) \mid \mathrm{ZnSe}]$ and [ZnSe|ITO-2(20.13 nm)|ZnSe] calculated by TMM and fitted by TCMT are shown in Fig. 5(d).

It is evident that both methods yield the same CPA wavelengths at 1429 and $1594 \mathrm{~nm}$. The broad CPA spectrum of [ZnSe|ITO-1|ITO-2|ZnSe] that was calculated by the TMM was also fitted by the sum of two Lorentzian spectra using Eq. (S15) in Supplementary Note B. We then obtained an equivalent CPA spectrum for [ZnSe|ITO$1|\mathrm{ITO}-2| \mathrm{ZnSe}$, which is slightly narrower than the simple summation of the two spectra and which corresponds closely to that calculated by the TMM. Then, we determined the intrinsic and radiative damping constants of [ZnSe|ITO-1|ITO-2 $\mid \mathrm{ZnSe}]$ structure to be $\Gamma_{i}=\Gamma_{r}=8.1 \times 10^{13} \mathrm{rad} / \mathrm{s}$ in the broad CPA spectrum, indicating that the broadband ENZ CPA is the coupling of the two resonant CPAs in the ENZ ITO layers.

We also numerically simulated the same [ZnSe|ITO-1|ITO-2|ZnSe] structure, and verified that our analytic results agree well with the finite-difference time domain (FDTD) simulations. We show field profiles for our CPA structure too. For more details on numerical simulations, see Fig. S1 in Supplementary Information.

Multi-wavelength All-optical Switching. Finally, we investigate the optical switching of the broadband CPA of [ZnSe|ITO-1|ITO-2|ZnSe]; $P_{\text {out } 1}$ and $P_{\text {out } 2}$ are shown on 2 D contour maps as a function of the 
(a)

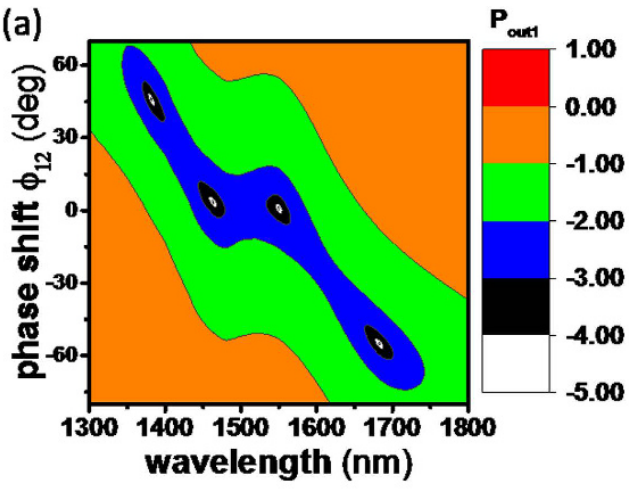

(c)

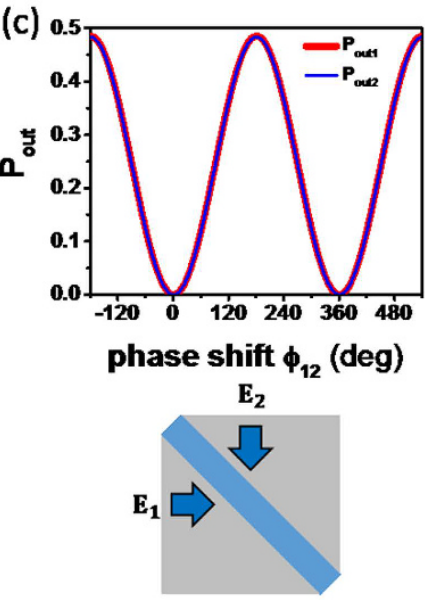

(d) 0.

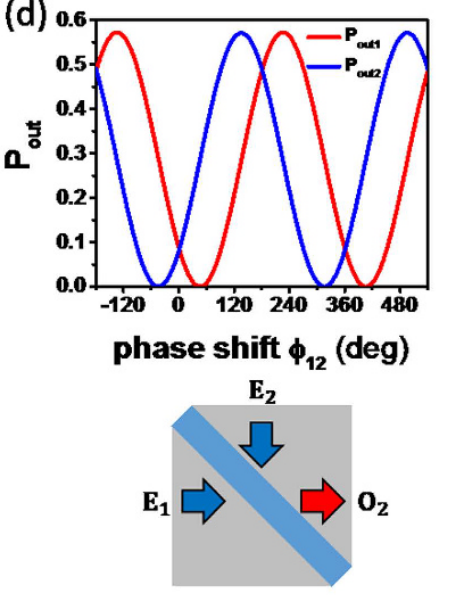

(b)

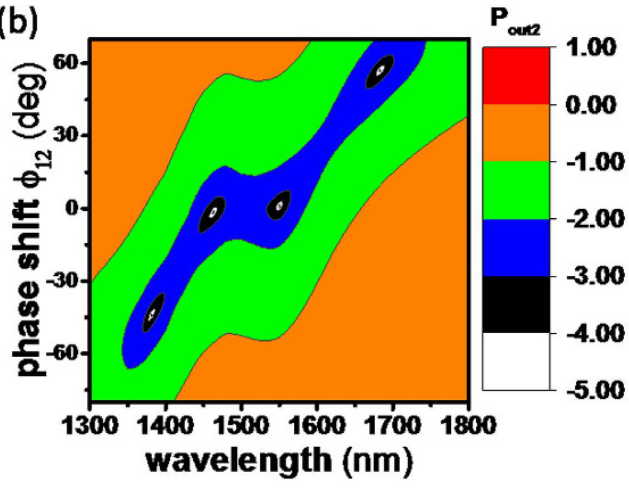

(e)

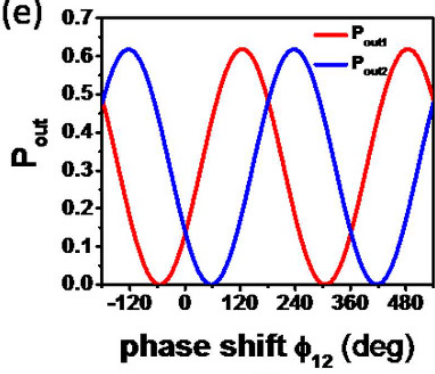

$\mathbf{E}_{\mathbf{2}}$

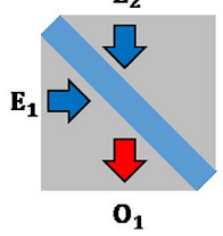

Figure 6. $2 \mathrm{D}$ contour maps of $(\mathbf{a}) \log \left(P_{\text {out } 1}\right)$ and $(\mathbf{b}) \log \left(P_{\text {out } 2}\right)$ as a function of $\varphi_{12}$. The color map is on the $\log$ scale. Optical switching of $P_{\text {out } 1}$ and $P_{\text {out } 2}$ at (c) $1550 \mathrm{~nm},(\mathbf{d}) 1381 \mathrm{~nm}$, and (e) $1683 \mathrm{~nm}$ as a function of $\varphi_{12}$.

\begin{tabular}{|c|c|c|c|c|}
\hline & $\begin{array}{l}\text { Wavelength } \\
(\mathrm{nm})\end{array}$ & $\begin{array}{l}\text { Phase shift } \\
\left(\phi_{12}, \text { degree }\right)\end{array}$ & $P_{\text {out } 1}($ Port 1) & $P_{\text {out } 2}($ Port 2) \\
\hline \multirow{4}{*}{ Single- wavelength CPA } & \multirow{2}{*}{1381} & $-44^{\circ}$ & $\begin{array}{c}\text { OFF } \\
\left(P_{\text {out } 1}=0.29, \Delta_{1}=-90^{\circ}\right)\end{array}$ & $\begin{array}{c}\text { ON } \\
\left(P_{\text {out } 2}=0, \Delta_{2}=0^{\circ}\right)\end{array}$ \\
\hline & & $46^{\circ}$ & $\begin{array}{c}\text { ON } \\
\left(P_{\text {out } 1}=0, \Delta_{1}=0^{\circ}\right)\end{array}$ & $\begin{array}{c}\text { OFF } \\
\left(P_{\text {out } 2}=0.29, \Delta_{2}=-90^{\circ}\right)\end{array}$ \\
\hline & \multirow{2}{*}{1683} & $-55^{\circ}$ & $\begin{array}{c}\text { ON } \\
\left(P_{\text {out } 1}=0, \Delta_{1}=0^{\circ}\right)\end{array}$ & $\begin{array}{c}\text { OFF } \\
\left(P_{\text {out } 2}=0.43, \Delta_{2}=112^{\circ}\right)\end{array}$ \\
\hline & & $57^{\circ}$ & $\begin{array}{c}\text { OFF } \\
\left(P_{\text {out } 1}=0.43, \Delta_{1}=112^{\circ}\right)\end{array}$ & $\begin{array}{c}\mathbf{O N} \\
\left(P_{\text {out } 2}=0, \Delta_{2}=0^{\circ}\right)\end{array}$ \\
\hline Broadband CPA & $1443-1576$ & $1^{\circ}$ & $\begin{array}{c}\text { ON } \\
\left(P_{\text {out } 1}=0, \Delta_{1}=0^{\circ}\right)\end{array}$ & $\begin{array}{c}\text { ON } \\
\left(P_{\text {out } 2}=0, \Delta_{2}=0^{\circ}\right)\end{array}$ \\
\hline
\end{tabular}

Table 2. Multi-wavelength optical switches in the broadband ENZ multilayer-ITO CPA device of [ZnSe|ITO-1|ITO-2|ZnSe] structure.

relative phase shift $\varphi_{12}$ and incident wavelength in Fig. 6(a,b), respectively. Firstly, broadband CPA greater than $99 \%$ absorptance is observed in the $1443 \sim 1576 \mathrm{~nm}$ wavelength range at $1^{\circ}$ phase shift (horizontal blue area, $O_{1}=O_{2}=0$, i.e. two-port CPA) in Fig. $6(\mathrm{a}, \mathrm{b})$; the bandwidth is $\sim 133 \mathrm{~nm}$ in spectrum and the phase shift range is $-5 \sim 7^{\circ}$. Secondly, the narrowband one-port CPAs are also evident: the one-port CPAs with $O_{1}=0$ are found at $\lambda=1381 \mathrm{~nm}, \varphi_{12}=46^{\circ}$ and $\lambda=1683 \mathrm{~nm}, \varphi_{12}=-55^{\circ}$ (tilted blue area) in Fig. 6(a), whereas the one-port CPAs with $O_{2}=0$ are evident at $\lambda=1381 \mathrm{~nm}, \varphi_{12}=-44^{\circ}$ and $\lambda=1683 \mathrm{~nm}, \varphi_{12}=57^{\circ}$ in Fig. $6(\mathrm{~b})$.

The two-port broadband CPA optical switching at $1550 \mathrm{~nm}$ is shown as a function of $\varphi_{12}$ in Fig. 6(c), and the "ON" state $\left(O_{1}=O_{2}=0\right)$ is apparent in both ports at $\varphi_{12}=1^{\circ}$. Similarly the broadband optical switching is evident at wavelengths of $1443 \sim 1576 \mathrm{~nm}$ at $\varphi_{12} \approx 1^{\circ}$. The optical switching of one-port CPA at $1381 \mathrm{~nm}$ and $1683 \mathrm{~nm}$ is shown in Fig. 6(d,e), respectively. At $1381 \mathrm{~nm}$, the "ON" state with $O_{1}=0$ appears at $\varphi_{12}=46^{\circ}$, whereas the "ON" state with $O_{2}=0$ appears at $\varphi_{12}=-44^{\circ}$. Similarly at $1683 \mathrm{~nm}$, the "ON" state with $O_{1}=0$ appears at $\varphi_{12}=-55^{\circ}$, whereas the "ON" state with $O_{2}=0$ appears at $\varphi_{12}=57^{\circ}$. Thus, the one-port optical switching of the narrowband $\mathrm{CPA}$ at $1381 \mathrm{~nm}$ and $1683 \mathrm{~nm}$ depends on the phase shift, and the two-port optical switching of the broadband 
CPA at $1443 \sim 1576 \mathrm{~nm}$ at $\varphi_{12}=1^{\circ}$. The multi-wavelength optical switching behaviors in narrowband and broadband [ZnSe|ITO-1|ITO-2|ZnSe] CPA device are summarized in Table 2.

We also investigated the incident angle dependence of the broadband CPA spectrum, which is shown in Fig. S2. As the incident angle increases from $44.5^{\circ}-49^{\circ}$, the bandwidth between two CPA dips at $1466 \mathrm{~nm}$ and $1550 \mathrm{~nm}$ narrows, keeping the center wavelength middle in the band, until a single, merged broad resonance appears.

\section{Discussion}

We want to clarify the difference between previous CPA proposals and our broadband CPA scheme. Previous CPA proposals were mostly based on nanostructured films. In those cases, elaborate multiple resonances in nanostructures may be employed to achieve broadband CPA. But, this can complicate the device structure and fabrication procedure. In contrast, we use simple, planar ENZ films, where optical properties can be easily controlled during the film growth (e.g. tuning film thicknesses and doping levels). Our approach simplifies the device fabrication and also facilitates the chip-scale integration with other optical components.

We notice that Ref. 8 also reported on CPA based on ultra-thin films. It studied two cases: Woltersdorff thickness and plasmon thickness. The former corresponds to a low-frequency region ( $\mathrm{DC}$ to $\sim 1 \mathrm{THz}$ ). The latter corresponds to the ENZ regime (i.e. $\operatorname{Re}(\varepsilon)=0$ ). Figure 6 in Ref. 8 shows strong absorption over a broad near-IR region in an ultra-thin tungsten film. But, as studied in Refs 23 and 32, normal-incidence perfect absorption in an ultra-thin film can happen only in a very lossy (large $\operatorname{Im}(\varepsilon)>1)$ film. It is hard to achieve this in other low-loss $(\operatorname{small} \operatorname{Im}(\varepsilon)<1)$ materials. However, in our CPA scheme, perfect absorption can occur in a low-loss, ultra-thin ENZ film, thanks to critical coupling at the ENZ wavelength ${ }^{23,24}$. Moreover, this can be easily extended to broadband CPA using ENZ multilayer films. Therefore, ENZ materials provide a very general, flexible platform for high-frequency, broadband CPA. We believe that many CPA devices can benefit from our approach.

In summary, we proposed the broadband ENZ CPA system based on tunable ITO thin films, and investigated its application to multi-wavelength all-optical switching in the near-IR region. We derived general CPA conditions first, using the scattering matrix and admittance matching methods separately, and demonstrated their equivalence for thin-film CPA. Then, we designed a [ZnSe|ITO-1|ITO-2|ZnSe] structure exhibiting the broadband CPA at $45^{\circ}$ incident angles in two ZnSe prisms. We found that the admittance matching method could extract not only the structural parameters, such as layer thicknesses and incident angles in media, but also input beam parameters, such as the power ratio and phase difference between two input beams. Finally, we analyzed multi-wavelength optical switching in our broadband ENZ CPA system. We can broaden the bandwidth further by increasing the number of ENZ layers. The same method can be also readily extended to other frequency ranges using different ENZ materials. Our proposal and theoretical analysis can provide design principles and guidelines for thin-film CPA devices, which can find various applications in optical switches, modulators, filters, sensors, and thermal emitters.

\section{References}

1. Chong, Y. D., Ge, L., Cao, H. \& Stone, A. D. Coherent Perfect Absorbers: Time-Reversed Lasers. Phys. Rev. Lett. 105, 053901 (2010).

2. Gmachl, C. F. Suckers for light. Nature 467, 37-39 (2010).

3. Noh, H., Chong, Y., Stone, A. D. \& Cao, H. Perfect coupling of light to surface plasmons by coherent absorption. Phys. Rev. Lett. 108, 186805 (2012).

4. Wan, W. et al. Time-Reversed Lasing and Interferometric Control of Absorption. Science 331, 889 (2011).

5. Zhang, J., MacDonald, K. F. \& Zheludev, N. I. Controlling light-with-light without nonlinearity. Light Sci. Appl. 1, 1-5 (2012).

6. Kang, M., Chong, Y. D., Wang, H.-T., Zhu, W. \& Premaratne, M. Critical route for coherent perfect absorption in a Fano resonance plasmonic system. Appl. Phys. Lett. 105, 131103 (2014).

7. Chen, T., Duan, S. \& Chen, Y. C. Electrodynamics analysis on coherent perfect absorber and phase-controlled optical switch. J. Opt. Soc. Am. A 29, 689-694 (2012).

8. Pu, M. et al. Ultrathin broadband nearly perfect absorber with symmetrical coherent illumination. Opt. Express 20, 2246-2254 (2012).

9. Dutta-Gupta, S., Martin, O. J. F., Gupta, S. D. \& Agarwal, G. S. Controllable coherent perfect absorption in a composite film. Opt. Express 20, 1330 (2012).

10. Zhang, J. et al. Coherent perfect absorption and transparency in a nanostructured graphene film. Opt. Express 22, 12524 (2014)

11. Fan, Y. et al. Tunable mid-infrared coherent perfect absorption in a graphene meta-surface. Sci. Rep. 5, 13956 (2015).

12. Li, S. et al. Broadband perfect absorption of ultrathin conductive films with coherent illumination: Superabsorption of microwave radiation. Phys. Rev. B. 91, 220301(R) (2015)

13. Rao, S. M., Heitz, J. J. .., Roger, T., Westerberger, N. \& Faccio, D. Coherent control of light interaction with graphene. Opt. Lett. 39, 5345-5347 (2014).

14. Engheta, N. Persuing near-zero response. Science 340, 286-287 (2013).

15. Jun, Y. C. et al. Epsilon-Near-Zero Strong Coupling in Metamaterial-Semiconductor Hybrid Structures. Nano Lett. 13, 5391-5396 (2013).

16. Campione, S., Brener, I. \& Marquier, F. Theory of epsilon-near-zero modes in ultrathin films. Phy. Rev. B 91, 121408(R) (2015).

17. Jin, Y., Xiao, S., Mortensen, N. A. \& He, S. Arbitrarily thin metamaterial structure for perfect absorption and giant magnification. Opt. Express 19, 11114-11119 (2011).

18. Feng, S. \& Halterman, K. Coherent perfect absorption in epsilon-near-zero metamaterials. Phys. Rev. B 86, 165103 (2012).

19. Luk, T. S. et al. Directional perfect absorption using deep subwavelength low permittivity films. Phys. Rev. B 90, 085411 (2014).

20. Luk, T. S. et al. Enhanced third harmonic generation from the epsilon-near-zero modes of ultrathin films. Appl. Phys. Lett. 106, 151103 (2015).

21. Park, J. Kang, J.-H., Liu, X. \& Brongersma, M. L. Electrically tunable epsilon-near-zero (ENZ) metal film absorbers. Sci. Rep. 5, 15754 (2015).

22. Ndiaye, C., Lemarchand, F., Zerrad, M., Ausserre, D. \& Amra, C. Optimal design for 100\% absorption and maximum field enhancement in thin-film multilayers at resonances under total reflection. Appl. Opt. 50, C382-C387 (2011).

23. Badsha, M. A., Jun, Y. C. \& Hwangbo, C. K. Admittance matching analysis of perfect absorption in unpattern thin films. Opt. Comm. 332, 206-213 (2014).

24. Yoon, J. et al. Broadband Epsilon-Near-Zero Perfect Absorption in the Near-Infrared. Sci. Rep. 5, 12788 (2015).

25. Collin, S. Nanostructure arrays in free-space: optical properties and applications. Rep. Prog. Phys. 77, 126402 (2014). 
26. Macleod, H. A. In Thin-Film Optical Filters 4th edn, Ch. 2, 42-61 (CRC Press, 2010).

27. Yeh, P. In Optical Waves in Layered Media, Ch. 5, 112-114 (John Wiley \& Sons, 2005).

28. Macleod, H. A. Optical absorption, Part 2. Bulletin (Society of Vacuum Coaters, Issue Fall) 13, 28-33 (2013).

29. Optical Constants of Electronic Materials and Transparent Conductors (280-2500 nm): URL https://windows.lbl.gov/materials/ chromogenics/N\&K-coverpage.html (Date of access: 28/12/2015).

30. Essential Macleod Thin Film Software (2015). Thin Film Center Inc, Tucson, AZ, USA. URL http://www.thinfilmcenter.com/.

31. Sun, L. \& Yu, K. W. Strategy for designing broadband epsilon-near-zero metamaterials. J. Opt. Soc. Am. B 29, 984-989 (2012).

32. Park, J., Kim, S. J. \& Brongersma, M. L. Condition for unity absorption in an ultrathin and highly lossy film in a Gires-Tournois interferometer configuration. Opt. Lett. 40, 1960-1963 (2015).

\section{Acknowledgements}

This work was supported by the Basic Science Research Program through a National Research Foundation of Korea grant funded by the Ministry of Education (NRF 2013R1A1A2007348). YCJ acknowledges the support from NRF grants (No. 2015001948, NRF-2014R1A1A2054108).

\section{Author Contributions}

T.Y.K., M.A.B., J.Y. and S.Y.L. performed simulations and modeling. Y.C.J. and C.K.H. conceived the idea and wrote the manuscript. C.K.H. supervised all aspect of the project. All authors reviewed the manuscript.

\section{Additional Information}

Supplementary information accompanies this paper at http://www.nature.com/srep

Competing financial interests: The authors declare no competing financial interests.

How to cite this article: Kim, T. Y. et al. General Strategy for Broadband Coherent Perfect Absorption and Multi-wavelength All-optical Switching Based on Epsilon-Near-Zero Multilayer Films. Sci. Rep. 6, 22941; doi: 10.1038/srep22941 (2016).

(c) (1) This work is licensed under a Creative Commons Attribution 4.0 International License. The images or other third party material in this article are included in the article's Creative Commons license, unless indicated otherwise in the credit line; if the material is not included under the Creative Commons license, users will need to obtain permission from the license holder to reproduce the material. To view a copy of this license, visit http://creativecommons.org/licenses/by/4.0/ 